\title{
Les représentations de l'animal et du bien-être animal par les éleveurs français
}

\author{
A.C. DOCKÈS, F. KLING-EVEILLARD \\ Institut de l'Elevage, 149 rue de Bercy, F-75595 Paris, France \\ Courriel : anne-charlotte.dockes@inst-elevage.asso.fr
}

\begin{abstract}
Dans un contexte où les éleveurs perçoivent une interrogation des autres acteurs de la société sur leurs pratiques vis-à-vis du bien-être animal, nous nous intéressons au point de vue de l'éleveur, acteur essentiel du bien-être des animaux. L'enjeu est d'une part de faciliter le dialogue entre les éleveurs et le reste de la société, et d'autre part d'éclairer et d'enrichir les formations destinées aux éleveurs eux-mêmes et aux intervenants qui les accompagnent.
\end{abstract}

\section{1 / Le lien entre attitudes, comportements des éle- veurs et bien-être des ani- maux a été largement démontré}

Jusqu'à présent, les travaux conduits sur le bien-être animal, conçus pour répondre à la fois aux attentes croissantes de la société sur ce thème et aux préoccupations éthiques des acteurs de l'élevage, ont principalement pris en compte les points de vue de la société d'une part, et de l'animal d'autre part. Nous nous attachons dans cet article à privilégier le point de vue de l'éleveur. En effet, de nombreux travaux réalisés depuis une vingtaine d'années montrent clairement qu'il existe un faisceau de corrélations positives entre les attitudes des personnes qui s'occupent des animaux d'élevage, leurs comportements vis-à-vis de ces animaux, l'état de bien-être des animaux et leurs performances zootechniques (voir notamment les travaux réalisés ou synthétisés par Hemsworth 2003).

Ainsi, les soigneurs qui considèrent que les porcs sont des animaux intelligents (Hemsworth et Coleman 1998), comme les éleveurs qui estiment que les veaux sont des êtres sensibles (Lensink 2000), auront un comportement plus doux vis-à-vis de leurs ani- maux. Ceux-ci auront moins peur de l'homme, seront en meilleure santé, et/ou auront une meilleure croissance.

Boivin et al (2003) insistent sur les conséquences positives d'un comportement attentif de l'homme également pour sa propre sécurité, pour la maîtrise technique de son élevage (Bochet et al 1983) et plus généralement pour son confort, sa satisfaction au travail (Hemsworth 2003). Reprenant la conception de Larrère et Larrère (2001) qui considèrent l'élevage comme un contrat social entre l'homme et l'animal, Boivin et al (2003) expliquent que de mauvaises relations entre l'éleveur et ses animaux peuvent être une source de "rupture» de contrat, alors que des relations positives sont à l'inverse un moyen de «matérialiser» ce contrat. Porcher (2001) a d'ailleurs étudié les difficultés dans l'exercice de leur métier ressenties par les éleveurs de porcs qui ne sont pas satisfaits, d'un point de vue éthique, des conditions de vie qu'ils procurent à leurs animaux ; réciproquement, elle a mis en évidence qu'une bonne relation de communication avec l'animal est source de satisfaction professionnelle.

Par ailleurs, le point de vue de l'éleveur sur l'animal d'élevage est fondamentalement différent de celui que lui porte le citoyen non éleveur. Veissier et Chambres (1999) ont par exemple montré que ces derniers personnalisaient largement l'animal familier (comme le chien) et rapprochaient l'animal d'élevage (comme le veau) de la viande. Pour les éleveurs, les deux types d'animaux sont plus proches l'un de l'autre, sans être pour autant identiques.

Une meilleure compréhension des attitudes des éleveurs et de leur diversité vise principalement deux objectifs. Elle fournit d'une part des éléments pour améliorer les actions de formation et de conseil destinées aux éleveurs. Il s'agira par exemple de renforcer leurs connaissances de l'animal pour leur permettre de faire évoluer leur propres attitudes et pratiques. Elle peut permettre d'autre part de favoriser le dialogue des éleveurs avec le reste de la société.

\section{2 / Des travaux spécifiques sur la représentation des animaux par les éleveurs et leur perception des attentes sociétales en matière de bien-être animal}

Les représentations sociales des éleveurs concernant leur métier, l'animal et le bien-être animal constituent notre objet d'étude. Nous considérerons les représentations au sens de Jodelet (1989) comme «une forme de connaissance, socialement élaborée et partagée, ayant une visée pratique», les représentations pouvant conduire à l'existence de normes sociales. Pour appréhender ces représentations, nous avons utilisé les méthodes d'entretiens semi-directifs approfondis, individuels ou collectifs (Blanchet et Gotman 1992), issues des démarches sociologiques et décrites dans Kling-Eveillard (2001). Les entretiens semi-directifs laissent à la personne enquêtée la possibilité d'une part d'exprimer son point de vue en réponse à des questions ouvertes très larges, et d'autre part d'organiser son discours en abordant les différents thèmes dans l'ordre qu'elle souhaite. Il s'agit d'enquêtes qualitatives, ayant un souci de prise en compte de la diversité des systèmes d'élevage et des points de vue, mais sans représentativité statistique. Nous avons conduit une série d'enquêtes dans les années 2000-2001, dans le cadre d'un programme de recherche financé par l'Association de Coordination Technique Agricole (ACTA) 
pour comprendre les représentations des éleveurs vis-à-vis de l'animal et du bien-être animal (Dockès et Kling 2006).

Ces enquêtes nous ont permis de rencontrer en France 28 éleveurs de porcins, 29 éleveurs de bovins et 28 éleveurs de volailles, dans des régions et des systèmes de production très diversifiés. Nous avons analysé leurs discours mais n'avons pas observé directement leurs comportements envers les animaux, ni l'état de bien-être de ceux-ci.

L'analyse de contenu est définie par Ghiglione et Mathalon (1998) comme l'analyse des propos tenus par les personnes interrogées, par rapport aux objectifs qui ont motivé leur recueil. La méthode d'analyse que nous avons utilisée consiste à répartir le contenu des propos recueillis selon des catégories thématiques et des catégories d'analyse (rubriques selon lesquelles chaque thème peut être analysé). Nous réalisons ensuite des synthèses thématiques (ce que disent les éleveurs sur chaque thème) et typologiques (quels sont les principaux groupes d'éleveurs qui se distinguent par leurs discours et leurs attitudes vis-à-vis de l'animal et du bien-être animal).

Les résultats présentés ci-dessous proviennent de nos propres travaux, éclairés par les résultats de la bibliographie.

\section{1 / Quelques points communs au plus grand nombre d'éle- veurs}

Un certain nombre d'éléments de discours sont communs à tous les profils d'éleveurs que nous avons rencontrés. Les éleveurs expriment tout d'abord le fait que leur relation aux animaux est d'ordre professionnel. Selon les personnes interrogées, être éleveur est un métier qui consiste à gagner sa vie avec les animaux. La relation à l'animal domestique n'est pas identique à la relation que les éleveurs peuvent entretenir avec leurs animaux familiers, ce qui est cohérent avec les résultats de Veissier et Chambres (1999).

Ensuite, être éleveur exige un rapport quotidien aux animaux. Les éleveurs doivent être en contact, souvent physique, avec eux. Ce contact peut être un plaisir ou une source de difficulté, voire de crainte, mais il est toujours présent. On peut faire l'hypothèse, à la suite des travaux de Boivin et al (2003) d'un lien fort entre la crainte que l'éleveur éprouve vis-à-vis de ses animaux, son comportement à leur égard, et les comportements dangereux des animaux. Le fait de travailler avec du vivant implique chez les éleveurs un engagement personnel fort, et un sentiment de responsabilité vis-à-vis de leurs animaux que tous considèrent comme des êtres sensibles, susceptibles de souffrir. Les éleveurs sont ainsi toujours affectés par les accidents sanitaires de leur troupeau.

Tous les éleveurs déclarent passer du temps à observer, à surveiller si tout se déroule d'une façon qu'ils jugent normale. Savoir observer les animaux est aux yeux de presque tous une des caractéristiques essentielles du bon éleveur. Un éleveur de bovins naisseurengraisseur explique ainsi : "Le bon éleveur, c'est celui qui passe du temps... même à ne rien faire. C'est-àdire à observer, à détecter si une bête tousse ou a une diarrhée, si elle boîte, toutes les anomalies de comportement».

Lensink (2000) aboutit, dans ses travaux sur les veaux de boucherie et leurs éleveurs, à une définition du «bon éleveur» extrêmement proche de celle que nous ont donnée les éleveurs : de bonnes connaissances techniques et organisationnelles ; une connaissance des animaux et de leurs besoins ; la patience, l'attachement ou la compassion visà-vis des animaux ; une bonne capacité d'observation et de surveillance.

\section{2 / Quatre profils principaux, en fonction de la proximité de l'éleveur avec l'animal}

Au-delà de ces considérations transversales, nous avons identifié, à partir de l'analyse du contenu de leurs discours, quatre profils principaux d'atti-

Figure 1. Quatre types d'attitudes chez les éleveurs.

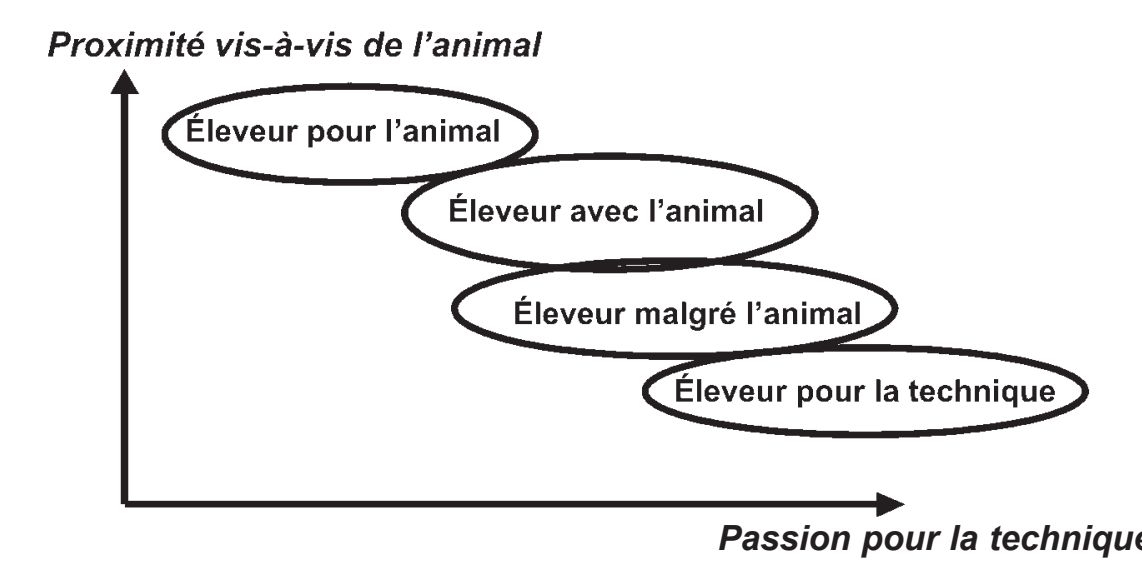

Passion pour la technique tudes chez les éleveurs, en fonction de leurs représentations de leur métier, de leurs animaux et du bien-être animal. On rencontre, dans chaque type, des éleveurs d'animaux de différentes espèces, un même éleveur pouvant d'ailleurs avoir des attitudes différentes vis-à-vis de chacun des types d'animaux qu'il élève (figure 1). Il est intéressant de noter qu'il n'existe que peu de liens entre les attitudes des éleveurs et leur profil sociologique (âge, formation...), ou leur système d'exploitation (à part des liens avec le type d'animaux élevés, que nous évoquerons ci-dessous). Enfin, étant donné le caractère qualitatif des travaux que nous avons conduits, nous ne sommes pas en mesure de fournir des éléments sur la représentativité statistique des différents profils. Tous étaient présents dans nos enquêtes, dans des proportions sensiblement équivalentes.

a) Eleveur pour l'animal : l'animal est au centre de la vie de l'éleveur

Les «éleveurs pour l'animal» n'auraient pas pu concevoir leur vie sans animaux et ne voient quasiment que les aspects positifs du métier. Ils ont une relation affective avec leurs animaux, qu'ils connaissent individuellement, et affichent une préférence pour certains, qu'ils ont parfois du mal à réformer. Ces éleveurs apprécient particulièrement les aspects de leur métier qui les mettent en contact avec l'animal, comme leur manipulation. Ils se situent dans une attitude résolument empathique par rapport à leurs animaux "Quand j'arrive dans le troupeau, la vache qui va se lever en s'étirant, c'est qu'elle est bien. Du coup, moi aussi je m'étire» (Eleveur de bovins, Nièvre). Ils considèrent enfin qu'il est légitime que la société exprime des attentes dans le domaine du bien-être, mais ne se sentent absolument pas mis en accusation. Ils sont plus nombreux à exprimer 
ce type d'attitude vis-à-vis des bovins, voire des porcins en ce qui concerne les animaux reproducteurs.

b) Eleveur avec l'animal : communiquer avec l'animal fait partie du métier

Les «éleveurs avec l'animal» pratiquent plutôt leur métier dans une continuité familiale. Ils voient des aspects positifs à leur métier (liberté, gestion du vivant) et des contraintes (temps, pénibilité, risque physique, aléas). Selon eux, l'animal est un être sensible avec lequel ils communiquent volontiers, mais ils ne s'attachent pas à leurs animaux pris individuellement. Ils apprécient les aspects techniques du métier d'éleveur (1'alimentation, la génétique, la production,...). Ils cherchent à être bien équipés pour la manipulation des animaux. Ils connaissent l'importance de la surveillance, de l'observation des animaux, mais cherchent à ne pas y passer trop de temps. Il est important à leurs yeux de communiquer avec le grand public, en expliquant que les éleveurs font bien les choses. Certains sont prêts à évoluer pour répondre aux attentes sociétales. Ces attitudes sont plus fréquentes vis-àvis des bovins et porcins, voire dans les élevages de volailles fermières.

A la suite des travaux conduits par les éthologues (synthétisés par Hemsworth 2003 et Boivin et al 2003), nous faisons l'hypothèse que les «éleveurs pour l'animal» et les «éleveurs avec l'animal», qui ont un discours «plutôt» à «très» positif sur leurs animaux, ont probablement des comportements relativement doux et empathiques dans leurs élevages, et en conséquence des réactions positives de leurs animaux. Cela peut expliquer leur «confort» au travail et le plaisir évident qu'ils y prennent.

c) Eleveur malgré l'animal : l'animal est une contrainte du métier d'éleveur

Les «éleveurs malgré l'animal» exercent leur métier par continuité familiale et ont choisi leur production pour des raisons économiques plus que par passion. Ils insistent sur les difficultés du métier comme sur son intérêt, en particulier sur les satisfactions liées à la productivité technique et à l'autonomie d'organisation. La communication avec l'animal est instrumentalisée. C'est une nécessité technique mais les éleveurs de ce groupe cherchent à la limiter et n'y prennent pas plaisir, certains craignent même le contact avec les animaux et notamment leur manipulation. Les attentes de la société sont perçues comme légitimes dans la mesure où elles confortent un choix de système (par exemple pour les éleveurs de volailles en plein air), et comme illégitimes et agressives dans les autres situations (par exemple pour un élevage de poules en cages). On rencontre ce type d'attitude dans toutes les productions, mais elles sont assez rares chez les éleveurs de bovins.

d) Eleveur pour la technique : les techniques de l'élevage sont passionnantes

Les «éleveurs pour la technique» ne considèrent pas que la relation à l'animal est essentielle dans leur métier. Il s'agit d'éleveurs passionnés par les aspects techniques de leur profession et notamment pour la gestion du vivant dans sa complexité, mais ils ne communiquent pas avec leurs animaux. Ils aiment ce qui touche à la technique et à la mécanique, mais aussi à l'observation et à la surveillance du troupeau ou du lot pour optimiser son fonctionnement. La mort de l'animal n'est vécue comme un problème que lorsqu'il s'agit d'un accident majeur. Ils confèrent essentiellement aux animaux des besoins physiologiques et considèrent qu'ils les satisfont. Ils s'estiment agressés par ce qu'ils perçoivent comme les demandes de la société qu'ils jugent parfois incompatibles avec la gestion économique de leurs exploitations. Ils sont plus nombreux dans le secteur de la volaille, mais présents dans toutes les productions.

Toujours à titre d'hypothèse, le discours plus «craintif» ou «mécaniste» des «éleveurs malgré l'animal» et «éleveurs pour la technique» vis-à-vis de leurs animaux est probablement à mettre en lien avec des comportements plus «indifférents» et/ou «agressifs» vis-àvis des animaux. Cela peut être à l'origine de réactions de peur, et d'agression chez les plus gros animaux, et expliquer des situations qui soient effectivement dangereuses pour l'homme (en particulier dans le profil «éleveur malgré l'animal»).

\section{3 / Les attitudes des éle- veurs vis-à-vis du bien-être animal et de sa réglementa- tion, dépendent des filières et de leur participation à des démarches de qualité}

Toujours en utilisant la méthode des entretiens semi-directifs, nous avons réalisé une seconde série d'enquêtes au cours des années 2004-2006 dans le cadre du projet Welfare Quality ${ }^{\circledR}$. Ces enquêtes avaient pour objectif d'identifier les freins et motivations des éleveurs vis-à-vis de démarches de qualité comportant un volet bien-être et de comprendre quelle perception les éleveurs avaient de la réglementation. Nous avons réalisé des enquêtes auprès d'une soixantaine d'éleveurs de porcins (Souquet et al 2006) et d'autant d'éleveurs de bovins (Bertin et al 2006). Une série d'enquêtes est en cours dans les filières de volailles. Des investigations parallèles ont été conduites dans 6 pays européens : la France, les PaysBas, 1'Italie, la Norvège, la Suède et la Grande Bretagne et partiellement synthétisées (Bock et Leuven 2005, Bock et al 2006). Les éléments suivants se dégagent des premières analyses transversales portant sur 360 éleveurs de porcs :

- globalement, les éleveurs qui sont dans des démarches de qualité avec des niveaux d'exigence relativement limités en matière de bien-être (identiques à la réglementation, ou ne bouleversant pas les systèmes d'élevage) se différencient peu les uns des autres, ils associent avant tout le bien-être à la satisfaction des besoins physiologiques des animaux et lient le bien-être aux performances zootechniques et économiques de leurs exploitations. Ils estiment souvent que leur production souffre d'une mauvaise image dans l'opinion publique. Ils se rapprochent de fait des troisième et quatrième profils d'attitude décrits plus haut ;

- les éleveurs impliqués dans des démarches exigeantes (démarches spécifiques bien-être, agriculture biologique, labels Rouges fermiers par exemple) mettent d'abord en avant la possibilité pour l'animal d'exprimer ses comportements naturels, autour des notions de liberté et de confort, dans une approche qui met en avant l'éthique de leur métier. Bien sûr les préoccupations économiques sont également très présentes, voire prioritaires, dans les justifications de leur implication dans une démarche de qualité. Ils estiment bénéficier d'une bonne image pour leurs produits et leurs modes de production. Ils sont souvent plus proches des deux premiers profils d'attitudes décrits plus haut.

Le niveau des réglementations concernant le bien-être est assez différent selon les filières et selon les pays (Menghi et Kling-Eveillard 2005). Les pays scandinaves sont caractérisés par un niveau de réglementation élevé, et des filières peu segmentées. Le bien- 
être semble considéré comme une question éthique et un niveau important est attendu de tous les éleveurs. Dans les pays latins à l'inverse, si les démarches de qualité sont nombreuses et diverses, les réglementations «bienêtre» sont quant à elles calquées sur le niveau européen.

Au niveau européen, les filières porcines, poules pondeuses et veaux de boucherie sont concernées par des réglementations précises, alors que l'ensemble des éleveurs doit suivre des préconisations générales. Nos enquêtes ont permis de constater que les éleveurs concernés par ces dernières (en production de gros bovins notamment) les connaissent peu et ont globalement l'impression de les respecter (Bertin et $a$ 1 2006), ils se sentent peu remis en cause par les réglementations concernant le bien-être animal. A l'inverse, les éleveurs de porcins qui doivent se conformer à de nouvelles règles les critiquent très largement. Ils craignent qu'elles ne remettent en cause leur équilibre économique, sans améliorer le bien-être de leurs animaux (Souquet et al 2006).

Il est intéressant de noter à partir de l'exemple des veaux de boucherie (Bertin et al 2006) que la mise en place de normes réglementaires nouvelles a parfois été l'occasion d'une évolution profonde dans les représentations de leurs métiers par les éleveurs. Ainsi les éleveurs de veaux de boucherie qui ont dû mettre leurs bâtiments et leurs façons de produire aux normes se déclarent globalement satisfaits des changements induits, pour leurs animaux comme pour eux mêmes. Ceux qui sont allés le plus loin, installant leurs veaux en grands groupes sur paille, nous ont d'ailleurs plusieurs fois souligné que le changement de système s'était accompagné à leurs yeux d'un changement complet de métier. Ils sont passés d'un statut d'ouvrier à un statut d'éleveur. En changeant de système ils ont le sentiment d'avoir mieux pris en compte ce que la société attendait d'eux, dont le bien-être de leurs animaux, et se sentent mieux dans leur métier. «J'ai choisi ce système (Distributeur d'Aliments Liquides, élevage sur paille), il permet de voir nos veaux. C'est un autre métier, complètement différent. Pendant 25 ans on était comme une machine. Le boulot c'était 4-5 heures à mettre du lait dans des seaux. Autant être avec les veaux pendant ce temps. Les veaux sont contents, c'est autre chose, une qualité de travail pour l'éleveur, comme pour les bêtes...
Et puis on est fiers d'ouvrir notre élevage, de montrer ce qu'on fait».

\section{4 / Discussion}

L'animal est au cœur de la définition de leur métier par la plupart des éleveurs. L'observation des animaux apparaît systématiquement dans leurs discours comme un élément essentiel, et c'est l'activité que la plupart des éleveurs préfèrent. Si le terme de bien-être animal n'est pas utilisé spontanément par les éleveurs, la nécessité d'être attentif à l'animal et le rejet des «mauvais traitements» sont contenus dans la définition qu'ils donnent de leur métier.

Le lien entre production et bien-être est souvent affirmé. Lorsqu'on leur pose la question, la quasi-totalité des éleveurs se reconnaît derrière l'affirmation selon laquelle le bien-être des animaux est assuré lorsque ceux-ci ont de bons niveaux de performance technique. Cette affirmation apparaît dans le discours des éleveurs comme une norme que l'on évoque sans la discuter, sans s'interroger sur sa pertinence, et que l'on utilise en particulier lorsque l'on se sent remis en question, comme «preuve» que l'on fait bien (Sens et Soriano 1998). Un nombre important d'éleveurs, cependant, ne pose pas spontanément le problème du bien-être en ces termes, mais du point de vue de l'animal lui même.

Les représentations que les éleveurs ont de leurs animaux sont liées à leurs conceptions du statut de l'animal (Burgat 1996, Larrère et Larrère 1997). Est-il un être sensible ou une machine ? Doit-il être libre ou instrumentalisé par l'homme pour son usage ? Aucun éleveur ne conteste que l'animal puisse souffrir, tous le définissent comme un être sensible. Mais son degré d'instrumentalisation est plus ou moins fort selon les éleveurs et selon les types d'élevage. D'après leurs discours, l'animal peut être analysé autour de trois pôles qui ne sont pas sans rappeler les profils d'éleveurs que nous avons identifiés (cf. supra) :

- l'animal instrumentalisé, l'éleveur porte essentiellement attention à ses fonctions de reproduction et à ses besoins physiologiques. Cette attitude est plus fréquente lorsque les animaux sont élevés dans de grands groupes homogènes (lots d'animaux), et qu'ils restent peu de temps dans l'élevage. Les «éleveurs malgré l'animal» et «les éleveurs pour la technique» se rapprochent de cette conception de l'animal ;
- l'animal communiquant, développant des relations d'échange positives avec l'homme, sources de satisfaction et de sécurité pour l'éleveur (relation fréquente avec des animaux en faible effectif et donc le plus souvent de grande taille, restant longtemps dans l'élevage). Les «éleveurs avec l'animal» se rattachent vraisemblablement à cette conception ;

- l'animal affectif, intégré dans une relation affective, d'attachement, à double sens avec l'homme. Cette conception semble particulièrement présente chez les «éleveurs pour l'animal», ne pouvant concevoir leur vie sans une relation directe et proche avec des animaux.

Tous les éleveurs pensent qu'un minimum de réglementation est nécessaire pour protéger les animaux contre les mauvais traitements et les abus. Audelà, les visions sont relativement contrastées et un lien ambivalent se dégage entre réglementation et perception du bien-être :

- certains éleveurs, dans le secteur bovin qui n'est pas concerné par des normes spécifiques, ne connaissent pas l'existence de réglementations précises sur le bien-être animal. Ils ont globalement l'impression de bien faire, de ne pas être remis en question et sont dans l'ensemble satisfaits de l'image que véhicule leur activité dans la société ;

- d'autres sont mieux informés de l'existence de réglementations, comprennent que la société puisse exprimer des exigences sur la question du bienêtre animal, et soit se considèrent en situation favorable, soit se déclarent prêts à s'adapter ;

- d'autres encore ne sont pas contre le principe de réglementations, mais critiquent le bien fondé technique des mesures existantes ou se sentent particulièrement agressés par leur contenu. Il s'agit souvent d'éleveurs ayant dû faire face à des changements récents, ou en cours dans les productions qui les concernent (porcs, volailles, voire veaux de boucherie). Ils souffrent souvent de l'image de leur production dans la société et sont plutôt en situation défensive en matière de communication avec le grand public ;

- enfin, certains qui ont dû se conformer à des règles nouvelles y ont vu une occasion d'évoluer fortement dans leurs pratiques et dans la conception qu'ils ont de leur métier.

Ainsi, l'animal est au cœur de la définition du métier de l'éleveur. La diversité de représentations de l'animal 
explique différentes visions du métier, différents niveaux d'intérêt dans le métier, et probablement, mais cela mériterait des travaux complémentaires, différents types de comportements vis-à-vis des animaux.

\section{Conclusion}

En conclusion, les représentations du bien-être animal par les éleveurs avaient pour l'instant été peu analysées, les travaux sur le bien-être s'étant jusqu'à présent surtout centrés sur 1'animal, sur les pratiques concrètes des éleveurs ou les attentes de la société. Notre travail, qui met l'éleveur au centre du débat sur son métier et sa relation avec les animaux, apporte un éclairage complémentaire sur ce thème. La prise en compte des représentations des éleveurs permet de montrer la diversité des types de relations entre éleveurs et animaux, même vis-à-vis d'une même espèce. Elle fournit ainsi des informations utiles pour concevoir des outils de conseil adaptés aux attentes des éleveurs, mais aussi, des formations destinées aux intervenants en élevage. En effet, Hemsworth et Coleman (1998) comme Chupin et Sarignac (1998) ont montré que des formations destinées aux éleveurs, et comportant à la fois des apports de connaissances sur les perceptions de l'animal et des travaux pratiques sur les comportements en élevage, permettaient de modifier la vision qu'avait l'éleveur de ses animaux comme sa façon d'agir vis-à-vis d'eux, et améliorait ainsi sa sécurité et son confort au travail. Les formations mises en place à l'Institut de l'Elevage auprès des techniciens sur le bien-être animal reprennent ces principes et comportent des phases d'apport de connaissances sur les attitudes des éleveurs et des travaux en exploitation. Les éléments d'analyse recueillis sur les relations entre l'éleveur et l'animal, sur la représentation de l'animal, constituent également une base pour concevoir et mettre en œuvre une communication sur le métier d'éleveur, destinée au grand public. Il s'agit d'apporter au public des informations à la fois sur l'animal d'élevage et sur le métier d'éleveur et ses relations avec ses animaux. Les travaux en cours dans différents pays européens fourniront en outre une base de comparaison qui permettra aux éleveurs et aux acteurs du développement français de se situer dans un référentiel plus vaste.

\section{Références}

Bertin M., Kling-Eveillard F., Dockès AC., 2006. Les éleveurs de bovins parlent du bien-être animal dans les démarches qualité. Institut de l'Elevage, collection Résultats, Paris, France, $46 p$.

Blanchet A., Gotman A., 1992. L'enquête et ses méthodes : l'entretien. Nathan Université, Paris, France, 128p.

Bock B, van Leeuwen F., 2005. Sociopolitical and market developments. In: Animal welfare concerns. Roex J., Meile M. de. (Eds). Cardiff University, UK, Welfare Quality report, 1, 115149.

Bock B., van Huik M., 2006. Pig farmers and animal welfare. A study of pig producers beliefs, attitudes and behaviour of pig producers across Europe. Welfare Quality report, 1312, 42p.

Bochet N., Benoit A., Chupin J.M., Houdoy D., 1983. Sécurité et facilité du travail : la relation homme-animal. Annuel manuel pour l'éleveur de bovin. ITEB (Ed), Paris, France, 125p.

Boivin X., Le Neindre P., Boissy A., Lensink J., Trillat G., Veissier I., 2003. Eleveurs et grands herbivores : une relation à entretenir. INRA Prod. Anim., 16 (2), 101-115.

Burgat F., 1996. Animal mon prochain, Odile Jacob (Ed), Paris, France, 245p.

Chupin J.M., Sarignac C., 1998. How to train cattle breeders to handling of bovine? In:
Veissier I., Boissy A. (Eds), Proc. $32^{\text {th }}$ Int. Cong. Int. Soc. Appl. Ethol., Clermont Ferrand, France, 117.

Dockès A.C., Kling F., 2005. Les éleveurs de bovins nous parlent de leur métier et de leurs animaux. Fourrages, 184, 513-522.

Dockès A.C., Kling F., 2006. Farmers' and advisers' representations of animals and animal welfare. Liv. Sci., 103, 243-249.

Ghiglione R., Matalon B., 1998. Les enquêtes sociologiques. Théories et pratiques. Armand Colin (Ed), Paris, France, 128p.

Hemsworth P.H., 2003 Human-animal interactions in livestock production. Appl. Anim. Behav. Sci., 81, 185-198.

Hemsworth P.H., Coleman G.J., 1998. Human-animal interactions: the stockperson and the productivity and welfare of intensively farmed animals. CAB international (Ed), New York, USA, 158p.

Jodelet D., 1989, Les représentations sociales. PUF (Ed), Paris, France, 423p.

Kling-Eveillard F., 2001. L'étude de motivation pour prendre en compte les points de vue des agriculteurs, Lignes, Institut de l'Elevage.

Larrère C., Larrère R., 1997. Le contrat domestique. Courrier de l'environnement, INRA, 30, 5-17.
Larrère C., Larrère R., 2001. Animal rearing as a contract? J. Agric. Env. Ethics, 12, 51-58.

Lensink B.J., 2000. Le rôle de l'éleveur dans le bien-être et la production de veaux de boucherie. Thèse de doctorat de l'Université de Rennes I, France, 238p.

Menghi A., Kling-Eveillard F., 2005. Animal welfare regulations and animal welfare schemes in 6 european countries. XXI Cong. Eur. Soc. Rural Sociol., Hungary, $7 \mathrm{p}$.

Porcher J., 2001. L'élevage, un partage de sens entre hommes et animaux : intersubjectivité des relations entre éleveurs et animaux dans le travail. Thèse INA-PG, Septembre 2001, 301p.

Sens S., Soriano V., 1998. Elever pour tuer. Une approche socio-zootechnique. Ethnozootechnie, 61, 9-18.

Souquet C., Kling-Eveillard F., Dockès AC., 2006. Les éleveurs de porcins parlent du bienêtre animal dans les démarches qualité. Institut de l'Elevage, collection Résultats, 38p.

Veissier I., Chambres P., 1999. Pour une approche indirecte du bien-être animal : les représentations du veau et du chien chez des citadins, ruraux et éleveurs. In : L'Homme et l'Animal : un Débat de Société. A.P. Ouédraogo, P. Le Neindre (Eds), Collection Mieux Comprendre, INRA Editions, 129-141.

\section{Résumé}

Plusieurs travaux récents ont permis d'appréhender les représentations des éleveurs, vis-à-vis de l'animal et du bien-être animal. Ils mettent en évidence un certain nombre d'éléments communs à tous les éleveurs : la place centrale de l'animal dans la définition du métier, la relation professionnelle aux animaux, le rôle central de l'observation dans le métier d'éleveur... Ils montrent la diversité des représentations de l'élevage, des animaux d'élevage et des pratiques, en fonction du type d'animaux, de l'histoire personnelle des individus et des démarches qualité dans lesquelles ils sont engagés : degré d'affectivité et d'attachement aux animaux, vision éthique de leur métier, perception des besoins des animaux... Les résultats de ces projets ouvrent des perspectives pour la recherche et le développement agricole afin de concevoir des outils pour le conseil, ainsi que des formations de techniciens ou d'éleveurs. 


\begin{abstract}
Livestock farmers' perception of animals and animal welfare

A number of recent projects have studied the way livestock farmers perceive animals and animal welfare. Farmers share certain ideas: animals play a central role in their profession; their relationship with animals is professional; observation is an essential part of their work. However, these studies have also highlighted that the way farmers perceive livestock farming, animals and practices varies widely in terms of emotional attachment to the animals, their ethical view of the profession, the perception of the animals' needs, and the definition of animal welfare. This diversity depends on the farmer's background, on the type of animals kept and on the quality scheme in which the farmer might be involved. The results of these projects have opened up new perspectives in research and development in animal rearing, leading to the creation of diagnostic and advisory tools, and the organisation of training courses for farmers and agricultural technicians.
\end{abstract}

DOCKÈS A.C., KLING-EVEILLARD F., 2007. Les représentations de l'animal et du bien-être par les éleveurs français. INRA Prod. Anim., 20, 23-28. 